\title{
EDITORIAL \\ The next generation of powered exoskeleton use in spinal cord injury
}

\author{
George M. Ghobrial, MD, and Michael Y. Wang, MD \\ Department of Neurological Surgery, University of Miami Miller School of Medicine, Miami, Florida
}

$\mathrm{S}$ PINAL cord injury (SCI) still accounts for more than 10,000 new cases per year, with a prevalence of approximately 1 million people in North America.,8 Furthermore, the life span of patients surviving the initial injury is approaching that of unaffected individuals. That being the case, the societal costs of these injuries are disproportionately high, and treatments targeting secondary injury pathways have fallen short of the mark. Early results for techniques aiming to replace lost populations of cellular architecture in the CNS are promising in theory, but still in the nascent stages of clinical translation.

Using current technology, an engineering and mechanical solution may obviate the numerous scientific and societal hurdles that retard progress toward restoring function in patients with SCI. One fundamental problem is that individuals with SCI expend high levels of metabolic energy either ambulating in a brace, or while nonambulatory in a wheelchair. ${ }^{5}$ Accordingly, the use of powered robotic exoskeletons can decrease that energy use. Gait orthoses powered by a robotic exoskeleton provide an externally powered gait by using motorized joints, and are steadily becoming lighter and more efficient. ${ }^{6}$ In a recent systematic review of gait speed achieved using powered exoskeletons, Louie et al. found that patients with thoracic-level, motor-complete SCI could safely attain modest speeds, with variations depending on operator experience with exoskeletons and the level of neurological injury. ${ }^{4}$ Moreover, with the use of exoskeletons, clinical improvement in the patient with SCI becomes a function of incremental improvements in exoskeleton design and energy efficiency. Arguably, this paradigm is far more manageable when compared to the obstacles encountered with implementation of targeted-treatment therapies for primary SCI mechanisms.

Against that background, Grasmücke and coinvestigators present their experience with the use of the Hybrid Assistive Limb (HAL, Cyberdyne Inc.) exoskeleton, a mechanical device capable of locomotive gait assistance via electromyographic stimuli. ${ }^{3}$ This represents the latest generation in assistive mechanical exoskeletons, whereby neurological feedback drives exoskeleton use. The aim of this study was to examine functional outcomes with regard to rehabilitation in patients with chronic SCI, adding valuable prospective clinical data to the limited body of literature. ${ }^{1,2}$ In the 55 patients studied, the authors conclude that one can expect approximately a $50 \%$ relative improvement in endurance and gait speed, as compared in the same individual without an assistive exoskeleton. Although the study included a limited number and heterogeneous group of patients, the authors should be commended on their efforts in providing one of the largest clinical series to date with current exoskeleton use. At the present time, the HAL exoskeleton is a temporary training tool and not a permanent aid for daily ambulation. However, in the foreseeable future, small incremental improvements in exoskeleton mechanical efficiency will extend the daily duration of use.

The restoration of function to patients with SCI by using such technologies is multifold. These devices may offer the greatest advantages for patients with incomplete SCI, converting measurable but functionally insignificant motor function into true mobility and independence. The salutary effects of restoring motion to the skeleton and joints in terms of cardiovascular benefits, prevention of contractures, osteoclastic activity, and psychological health have yet to be measured. Finally, the effect of restoring function on the process of neurogenesis remains a distant but real possibility.

\section{References}

1. Aach M, Cruciger O, Sczesny-Kaiser M, Hoffken O, Meindl $\mathrm{R}$, Tegenthoff M, et al: Voluntary driven exoskeleton as a new tool for rehabilitation in chronic spinal cord injury: a pilot study. Spine J 14:2847-2853, 2014 
2. Cruciger O, Tegenthoff M, Schwenkreis P, Schildhauer TA, Aach M: Locomotion training using voluntary driven exoskeleton (HAL) in acute incomplete SCI. Neurology 83:474, 2014

3. Grasmücke D, Zieriacks A, Jansen O, Fisahn C, Sczesny-Kaiser M, Wessling M, et al: Against the odds: what to expect in rehabilitation of chronic spinal cord injury with a neurologically controlled Hybrid Assistive Limb exoskeleton. A subgroup analysis of 55 patients according to age and lesion level. Neurosurg Focus 42(5):E15, 2017

4. Louie DR, Eng JJ, Lam T: Gait speed using powered robotic exoskeletons after spinal cord injury: a systematic review and correlational study. J Neuroeng Rehabil 12:82, 2015

5. Massucci M, Brunetti G, Piperno R, Betti L, Franceschini M: Walking with the advanced reciprocating gait orthosis (ARGO) in thoracic paraplegic patients: energy expenditure and cardiorespiratory performance. Spinal Cord 36:223227,1998
6. Sale P, Franceschini M, Waldner A, Hesse S: Use of the robot assisted gait therapy in rehabilitation of patients with stroke and spinal cord injury. Eur J Phys Rehabil Med 48:111-121, 2012

7. Witiw CD, Fehlings MG: Acute spinal cord injury. J Spinal Disord Tech 28:202-210, 2015

8. Wyndaele M, Wyndaele JJ: Incidence, prevalence and epidemiology of spinal cord injury: what learns a worldwide literature survey? Spinal Cord 44:523-529, 2006

\section{Disclosures}

Dr. Wang is a consultant for as well as a patent holder with DePuy Spine. He is also a consultant for Aesculap Spine, K2M, and JoiMax. He owns stock in ISD. 